\title{
Clustering Within Quantum Mechanical Framework
}

\author{
Güleser K. Demir \\ Dokuz Eylül University, \\ Department of Electrical and Electronics Engineering, \\ TR35460, Izmir, Turkey, \\ demir@eee.deu.edu.tr
}

\begin{abstract}
We study clustering problem within quantum mechanical framework by utilizing the Schroedinger equation written for the lowest energy state. We extend the analysis of Horn and Gottlieb [1] by providing an explicit discussion of probability distribution within full quantum mechanical context and examine the clustering performances for various probability distribution functions with numerical experiments.
\end{abstract}

\section{Introduction}

Recently, a clustering method that extents the scale-space clustering by using quantum mechanical analogies has been proposed by Horn and Gottlieb [1. This approach is interesting in that they have viewed probability distribution function as the stationary-state eigenfunction of Schroedinger equation with potential energy $V(\boldsymbol{x})$, and thereby, they have shown that minima of the potential energy identify the clusters. In the scale-space or density-based clustering the clusters are defined as dense regions, and each dense region is separated from one another by low density regions [2, 3]. In the quantum mechanical framework, the dense regions are identified as the valley regions of the quantum potential as follows from the Schroedinger equation. As a result, we envisage the minima of the quantum potential as the center points of the clusters, and equipotential levels of the potential as cluster boundaries.

In this work we will extend the analysis of Horn and Gottlieb [1] by providing an explicit discussion of probability distribution within full quantum mechanical context. Furthermore, we compute the potential energy for each data point $\boldsymbol{x}_{i}$, and determine the clustering performance by identifying probability distribution with various nonlinear mapping functions used in pattern recognition. As a byproduct of the application of full quantum formalism, we also show how the probability distribution changes with time, though we do not provide a full numerical study of this point at this stage yet.

In the next section we provide the quantum mechanical formalism. In Section 3 we provide numerical study of proposed clustering method for various probability distributions. In Section 4 we conclude the work. 


\section{The Quantum Potential}

The dynamics of a quantum system is described by the Schroedinger equation

$$
i \frac{\partial}{\partial t} \psi(\boldsymbol{x}, t)=\left[-\frac{\gamma^{2}}{2} \nabla^{2}+V(\boldsymbol{x}, t)\right] \psi(\boldsymbol{x}, t)
$$

where $\psi(\boldsymbol{x}, t)$ is the wavefunction and $V(\boldsymbol{x}, t)$ is the potential energy of the system [4], 5]. It is convenient to parameterize the wavefunction as $\psi(\boldsymbol{x}, t)=$ $\sqrt{\rho(\boldsymbol{x}, t)} e^{i S(\boldsymbol{x}, t)}$ where $\rho(\boldsymbol{x}, t)$ and $S(\boldsymbol{x}, t)$ are real functions of $\boldsymbol{x}$ and time $t$. Replacement of this form of the wavefunction in the Schroedinger equation results in two coupled equations

$$
-\frac{\partial}{\partial t} S=V+\frac{\gamma^{2}}{2}(\nabla S)^{2}-Q, \quad-\frac{\partial}{\partial t} \rho=\gamma^{2} \nabla(\rho \nabla S)
$$

where $Q$ is the "quantum potential"

$$
Q(\boldsymbol{x}, t)=\frac{\gamma^{2}}{2} \frac{\nabla^{2} \sqrt{\rho(\boldsymbol{x}, t)}}{\sqrt{\rho(\boldsymbol{x}, t)}} .
$$

In quantum mechanics the modulus-squared of the wavefunction is the probability density of the system, that is, $\rho(\boldsymbol{x}, t)$ gives the probability to find the system within the differential volume $d^{D} \boldsymbol{x}$. The real function $S(\boldsymbol{x}, t)$ is the phase of the wavefunction. It does not contribute to the probability density rather it determines the current density of probability flow. Depending on the temporal properties of the system, one can consider the following special cases:

1. Stationary-state quantum system with homogeneous phase: In this case system evolves in time with a definite energy. In particular, $V(\boldsymbol{x}, t)$ and thus $\rho(\boldsymbol{x}, t)$ is independent of time; moreover, the phase function depends solely on time $S(\boldsymbol{x}, t)=-E t$ where $E$ is the total energy of the system (kinetic plus potential energy). Under these conditions (2) gives rise to a static potential

$$
V(\boldsymbol{x})=Q(\boldsymbol{x})-Q(\boldsymbol{x})_{\min }
$$

if $V(\boldsymbol{x})$ is chosen to vanish at its minimum, implying $Q(\boldsymbol{x})_{\min }=-E$.

2. Stationary-state quantum system with inhomogeneous phase: In this case the phase field is given by $S(\boldsymbol{x}, t)=-E t+W(\boldsymbol{x})$ where $W$ is independent of time. Then (2) give rise to

$$
\begin{aligned}
V(\boldsymbol{x}, t) & =Q(\boldsymbol{x}, t)-\frac{\gamma^{2}}{2}(\nabla W)^{2}-\left(Q(\boldsymbol{x}, t)-\frac{\gamma^{2}}{2}(\nabla W)^{2}\right)_{\text {min }} \\
\frac{\partial}{\partial t} \rho(\boldsymbol{x}, t) & =-\gamma^{2} \nabla(\rho(\boldsymbol{x}, t) \nabla W(\boldsymbol{x})) .
\end{aligned}
$$

It is clear that now $V(\boldsymbol{x}, t)$ is determined by both the quantum potential and the phase field. Moreover, the probability density evolves in time with a rate determined by the inhomogeneity of the phase field. 


\section{Data Clustering Using Quantum Potential}

We analyze the implications of the following probability distribution functions

$$
\begin{array}{ll}
\rho_{1}(\boldsymbol{x})=\sum_{i=1}^{N} e^{-\frac{\left(\boldsymbol{x}-\boldsymbol{x}_{i}\right)^{2}}{2 \gamma^{2}}}, & \rho_{2}(\boldsymbol{x})=\left|\sum_{i=1}^{N} e^{-\frac{\left(\boldsymbol{x}-\boldsymbol{x}_{i}\right)^{2}}{2 \gamma^{2}}}\right|^{2}, \\
\rho_{3}(\boldsymbol{x})=\left|\sum_{i=1}^{N} e^{-\frac{\left(\boldsymbol{x}-\boldsymbol{x}_{i}\right)^{2}}{2 \gamma^{2}}}\right|^{4}, & \rho_{4}(\boldsymbol{x})=\left|\sum_{i=1}^{N} \frac{1}{\left(\left(\boldsymbol{x}-\boldsymbol{x}_{i}\right)^{2}+\gamma^{2}\right)^{2}}\right|^{2},
\end{array}
$$

by using each of them in (4) where $N$ is the total number of points. Here, we emphasize that all of these $\rho$ functions have local property. Namely, larger the $\rho$ larger the number of points in the neighborhood (whose size is determined by $\gamma$ ) of $\boldsymbol{x}$. Therefore, they are capable of representing structural relationships of the data points. The main tool for probing the cluster centers is the minima of the potential energy. The quantum potentials corresponding to the probability distribution functions $\rho_{1}, \rho_{2}, \rho_{3}$ and $\rho_{4}$ are given respectively by

$$
\begin{aligned}
Q_{1}(\boldsymbol{x}) & =-\frac{D}{4}+\frac{1}{4 \gamma^{2}\left(\rho_{1}(\boldsymbol{x})\right)^{2}} \sum_{i=1}^{N}\left(\boldsymbol{x}-\boldsymbol{x}_{i}\right)^{2} e^{-\frac{\left(\boldsymbol{x}-\boldsymbol{x}_{i}\right)^{2}}{2 \gamma^{2}}} \\
& -\frac{1}{8 \gamma^{2}\left(\rho_{1}(\boldsymbol{x})\right)^{4}} \sum_{i=1}^{N} \sum_{j=1}^{N}\left(\boldsymbol{x}-\boldsymbol{x}_{i}\right) \cdot\left(\boldsymbol{x}-\boldsymbol{x}_{j}\right) e^{-\frac{1}{2 \gamma^{2}}\left[\left(\boldsymbol{x}-\boldsymbol{x}_{i}\right)^{2}+\left(\boldsymbol{x}-\boldsymbol{x}_{j}\right)^{2}\right]} \\
Q_{2}(\boldsymbol{x}) & =-\frac{D}{2}+\frac{1}{2 \gamma^{2} \rho_{2}(\boldsymbol{x})} \sum_{i=1}^{N}\left(\boldsymbol{x}-\boldsymbol{x}_{i}\right)^{2} e^{-\frac{\left(\boldsymbol{x}-\boldsymbol{x}_{i}\right)^{2}}{2 \gamma^{2}}} \\
Q_{3}(\boldsymbol{x}) & =-D+\frac{1}{\gamma^{2}\left(\rho_{3}(\boldsymbol{x})\right)^{1 / 2}} \sum_{i=1}^{N}\left(\boldsymbol{x}-\boldsymbol{x}_{i}\right)^{2} e^{-\frac{\left(\boldsymbol{x}-\boldsymbol{x}_{i}\right)^{2}}{2 \gamma^{2}}} \\
& -\frac{1}{\gamma^{2} \rho_{3}(\boldsymbol{x})} \sum_{i=1}^{N} \sum_{j=1}^{N}\left(\boldsymbol{x}-\boldsymbol{x}_{i}\right) \cdot\left(\boldsymbol{x}-\boldsymbol{x}_{j}\right) e^{-\frac{1}{2 \gamma^{2}}\left[\left(\boldsymbol{x}-\boldsymbol{x}_{i}\right)^{2}+\left(\boldsymbol{x}-\boldsymbol{x}_{j}\right)^{2}\right]} \\
Q_{4}(\boldsymbol{x}) & =\sum_{i=1}^{N} \frac{-4 \gamma^{2}\left(\gamma^{2}-2\left(\boldsymbol{x}-\boldsymbol{x}_{i}\right)^{2}\right)}{\left(\gamma^{2}+\left(\boldsymbol{x}-\boldsymbol{x}_{i}\right)^{2}\right)^{4}} .
\end{aligned}
$$

In what follows we will perform a numerical study of these potentials for the Leptograpsus crab data set which has also been used by Horn and Gottlieb [1. For the ease of comparison, we follow the same preprocessings applied in that study. This data set consists of four classes each containing 50 instances in a five-dimensional data space. Its dimension is reduced to two by using Principal Component Analysis (PCA) [7. While applying PCA, the data are projected onto the second and third eigenvectors of the correlation matrix of the data set and then the projections are normalized by dividing them by the square roots of the corresponding eigenvalues. In Figure 1 we depict the data and the tomographic map of the quantum potential divided by its maximum value for 


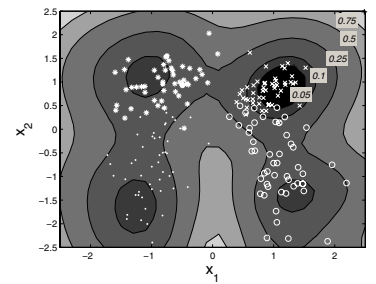

(a)

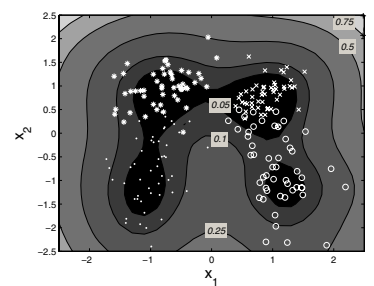

(c)

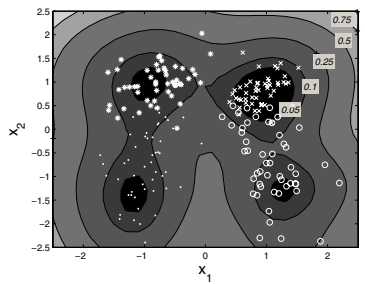

(b)

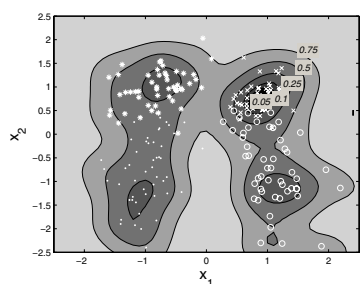

(d)

Fig. 1. Tomographic maps of the quantum potential for the probability distribution functions of (a) $\rho_{1}(\boldsymbol{x})$, (b) $\rho_{2}(\boldsymbol{x})$, (c) $\rho_{3}(\boldsymbol{x})$ and (d) $\rho_{4}(\boldsymbol{x})$

each of the probability distributions in (7). Figures 1 (a), (b) and (c) show the resulting maps for $Q_{1}, Q_{2}$, and $Q_{3}$ with $\gamma=1 / \sqrt{2}$, respectively. Figure 1 1 (d) shows the tomographic map for $Q_{4}$ with $\gamma=1$. Here, we illustrate the actual class of each data point with four different markers for enabling the visualization of clustering performance. The required four minima are seen clearly in the quantum potential energy distributions. Indeed, these minima correspond to four different cluster centers where equipotential lines are related to the cluster boundaries. One notes from the first three panels of Figure 1 that $Q_{1}$ exhibits a much sharper distribution than $Q_{2}$ and $Q_{3}$. This shows that for clustering purposes direct identification of quantum mechanical probability distribution $\rho(\boldsymbol{x})$ with the Parzen-window estimator works better than other alternatives. En passant, we note that the analysis of Horn and Gottlieb 1] corresponds to using $\rho_{2}$ for clustering, as one can see by rewriting quantum potential in (3) in their form. Figure 1.(d) is obtained for the inverse-quadratic probability distribution function, $\rho_{4}$. This potential shows a relatively different behavior than the Gaussian ones though it still clearly identifies the four cluster centers.

In this work, for each point in the data set we find an associated steepestascent trajectory which originates at the datum and ends in one of the local maxima. And then, a cluster is formed for all those points whose steepest-ascent trajectories converge to the same local minimum. Interesting enough, the same convergence behavior of trajectories can also be realized by the guiding trajectory driven by quantum potential in Bohmian quantum mechanics 8 . Figures 2 (a) and (b) show the resulting clustering assignments for the Gaussian probability function, $\rho_{2}$, and inverse quadratic probability function, $\rho_{4}$, respectively. 


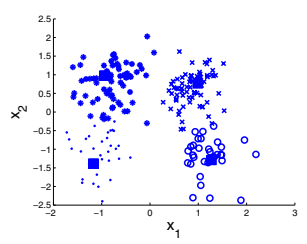

(a)

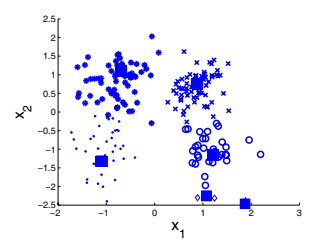

(b)

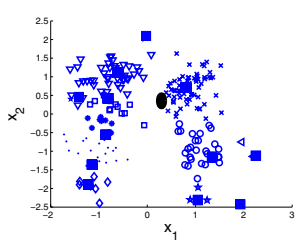

(c)

Fig. 2. Cluster assignments via gradient descent algorithm. Different markers show different clusters and filled square markers indicate cluster centers for (a) $\rho_{1}(\boldsymbol{x})$ with $\gamma=1$, (b) $\rho_{4}(\boldsymbol{x})$ with $\gamma=1$, (c) $\rho_{4}(\boldsymbol{x})$ with $\gamma=1 / \sqrt{2}$. Big filled ellipse in (c) shows the single cluster center for $\rho_{4}(\boldsymbol{x})$ with $\gamma=4$.

Additional two cluster centers are seen $x_{1} \cong 1$ and $x_{1} \cong 2$ in Figure 2 (b) due to the rather shallow local minima in these regions. We further examine the dependence of the clustering performance on the width parameter $\gamma$. For this we compare inverse-quadratic distribution function for $\gamma=1 / \sqrt{2}$ and $\gamma=4$. In Figure 2-(c) different markers show different clusters and filled squares show the cluster centers for $\gamma=1 / \sqrt{2}$. In the same sub-figure big filled ellipse shows the single cluster center for $\gamma=4$. Since the number of minima increases with decreasing $\gamma$ one needs to choose $\gamma$ judiciously for good clustering (which might cost a number of experiments). As a second experiment, we tested the proposed method on image compression application. The performances of the clustering by quantum potential and by the well-known k-means algorithm are compared for real images. 4-dimensional data points are obtained by choosing the block size as $2 \times 2$ in $64 \times 64$ images. The data vectors are normalized so that their means and variances are zero and one, respectively. For performing quantitative analyzes of the methods we use peak signal-to-noise ratio (PSNR). Table 1 shows the number of clusters for different values of $\gamma$ and corresponding PSNR values by using quantum potential and by using well-known k-means algorithm for the Lena image. Since $\gamma$ determines the size of neighboring region the number of clusters increases with the decrease of the $\gamma$.

The use of quantum mechanical framework for clustering purposes is a rather new approach. Though, as shown here, its performance is not as good as that of the k-means algorithm it opens up the possibility of quantum mechanical computations in neural systems and implementation of clustering at quantum level with significant profits on computational time and size.

Table 1. The number of clusters for different values of $\gamma$ and corresponding PSNR values by using quantum potential and by using k-means algorithm

\begin{tabular}{|c|c|c|c|}
\hline$\gamma$ & Number of Clusters & PSNR $_{Q}$ & PSNR $_{k-\text { means }}$ \\
\hline 0.5 & 24 & 58.82 & 60.37 \\
1 & 18 & 56.13 & 59.32 \\
2 & 7 & 54.01 & 57.21 \\
\hline
\end{tabular}




\section{Conclusion}

In this work we discussed the clustering problem within quantum mechanical context and gave experimental results. In the experiments, we have computed the potential energy for each data point $\boldsymbol{x}_{i}$, and depicted the clustering performances by using various probability distribution functions. We have assigned data points to the clusters by using gradient descent procedure.

Recently several researchers have studied on the idea of quantum mechanical computations in biological neural networks and proposed some associative neural/quantum network structures with possible implementations at quantum level [9], 10. Clustering in the quantum mechanical framework is an extension of these studies and it might become a basis for advanced research opportunities. For example, while representing the flow of probability distribution with the use of continuity equation one may trace and segment objects in motion analysis. Additionally, one may include the training data set into the calculations, again considering the minima of quantum potential as the regions which we want to minimize (such as training error).

\section{References}

1. D. Horn and A. Gottlieb, Algorithm for Data Clustering in Pattern Recognition Problems Based on Quantum Mechanics, Phys.Rev. Lett., 88, 2002.

2. S.J. Roberts, Non-parametric unsupervised cluster analysis, Pattern Recognition, 30,2, 261-272, 1997.

3. A. Hinneburg, D.A. Keim, An Efficient Approach to Clustering in Multimedia Databases with Noise, Proc. 4rd Int. Conf. on Knowledge Discovery and Data Mining, New York, AAAI Press, 1998.

4. S. Gasiorowicz, Quantum Physics, 3rd ed, (John Wiley and Sons, 2003).

5. E. Merzbacher, Quantum Mechanics, 3rd ed, (John Wiley and Sons, 1998).

6. B.D. Ripley, Ftp Archive, Department of Statistics, Univ. of Oxford, http://www.stats.ox.ac.uk/pub/PRNN/.

7. Morrison, D.F., Multivariate Statistical Methods , 2nd ed., (McGraw-Hill, New York, 1976).

8. Holland, Peter R. The Quantum Theory of Motion: An Account of the de BroglieBohm Causal Interpretation of Quantum Mechanics, (Cambridge: Cambridge U. Press, 1993).

9. R. Penrose, Shadows of the Mind, (Oxford University Press, 1994).

10. M. Perus, S.K. Dey, Quantum systems can realize content-addressable associative memory; Applied Math. Letters, 13, 8, 31-36, 2000. 